\title{
GREEDINESS OF THE HAAR SYSTEM IN REARRANGEMENT INVARIANT SPACES
}

\author{
P. WOJTASZCZYK \\ Institute for Applied Mathematics, Warsaw University \\ Banacha 2, 02-097 Warszawa, Poland \\ E-mail: pwojt@mimuw.edu.pl
}

1. Introduction. Greedy bases attracted some attention in recent years; mostly, I think, due to the fact that they can be studied both from concrete approximation theory point of view and from an abstract Banach space perspective. Let $\left(x_{n}\right)_{n \in \mathbb{N}}$ be a semi-normalised basis in a Banach space $X$. This means that $\left(x_{n}\right)_{n \in \mathbb{N}}$ is a Schauder basis in some fixed order (see [4]) and is semi-normalised, i.e. $0<\inf _{n \in \mathbb{N}}\left\|x_{n}\right\| \leq \sup _{n \in \mathbb{N}}\left\|x_{n}\right\|<\infty$. For an element $x \in X$ we define the error of the best $m$-term approximation as follows:

$$
\sigma_{m}(x)=\inf \left\{\left\|x-\sum_{n \in A} \alpha_{n} x_{n}\right\|\right\}
$$

where the inf is taken over all subsets $A \subset \mathbb{N}$ of cardinality at most $m$ and all possible scalars $\alpha_{n}$. We also define the greedy approximation of $x=\sum_{n} a_{n} x_{n} \in X$ as

$$
\mathcal{G}_{m}(x)=\sum_{n \in A} a_{n} x_{n}
$$

where $A \subset N$ is any set of cardinality $m$ chosen in such a way that $\left|a_{n}\right| \geq\left|a_{l}\right|$ whenever $n \in A$ and $l \notin A$. We say that a semi-normalised basis $\left(x_{n}\right)_{n \in \mathbb{N}}$ is greedy if there exists a constant $C$ such that for all $m=1,2, \ldots$ and all $x \in X$ we have

$$
\left\|x-\mathcal{G}_{m}(x)\right\| \leq C \sigma_{m}(x) .
$$

This notion evolved in the theory of non-linear approximation, see e.g. [10], [13]. Let us recall the following

Definition 1 ([7]). A basis $\left(x_{n}\right)_{n \in \mathbb{N}}$ in a Banach space $X$ is democratic if there exists a constant $C$ such that for all finite sets $A, B \subset \mathbb{N}$ of the same cardinality we have $\left\|\sum_{n \in A} x_{n}\right\| \leq C\left\|\sum_{n \in B} x_{n}\right\|$.

2000 Mathematics Subject Classification: Primary 41A46; Secondary 46B15.

This work was partially supported by the KBN grant 1 PO3A 03827 . The author gratefully acknowledges the support from the Foundation for Polish Science.

The paper is in final form and no version of it will be published elsewhere. 
The following characterisation of greedy bases was proved in [7].

THEOREM 1 ([7]). A semi-normalised basis $\left(x_{n}\right)_{n \in \mathbb{N}}$ in a Banach space $X$ is greedy if and only if it is a democratic and unconditional basis.

From this Theorem one easily obtains that if $\left(x_{n}\right)_{n \in \mathbb{N}}$ is a greedy basis and $\left(\lambda_{n}\right)_{n \in \mathbb{N}}$ is a sequence of numbers such that $0<\inf _{n \in \mathbb{N}}\left|\lambda_{n}\right| \leq \sup _{n \in \mathbb{N}}\left|\lambda_{n}\right|<\infty$ then $\left(\lambda_{n} x_{n}\right)_{n \in \mathbb{N}}$ is also a greedy basis.

From the approximation theory point of view the main example of a greedy basis is a Haar system in $L_{p}$ where $1<p<\infty$. Also good wavelet bases in $L_{p}$ are greedy. For a survey about greedy bases the reader may consult [14].

In this note we investigate if the Haar system is greedy in other rearrangement invariant spaces besides $L_{p}$. Surprisingly, the answer is no, see Theorem 2 . In subsequent sections we investigate Lorentz spaces $L_{p, q}$. First we characterise subsequences of the Haar system which are greedy in $L_{p, q}$, see Theorem 3 . We conclude this note with some properties of a greedy basis in $L_{2, q}$ if such a basis exists at all.

2. The Haar system in rearrangement invariant spaces. Let us recall that a rearrangement invariant space is a Banach space $(X,\|\|$.$) whose elements are (equivalence$ classes of) measurable functions on measure space $(\Omega, \mu)$ satisfying:

1. if $x \in X$ and $y$ is a measurable function such that $|y(\omega)| \leq|x(\omega)| \mu$-a.e. then $y \in X$ and $\|y\| \leq\|x\|$

2. if $x \in X$ and $y$ has the same distribution as $x$ (i.e. for all $\lambda \in \mathbb{R}, \mu(\{\omega \in \Omega: x(\omega)$ $<\lambda\})=\mu(\{\omega \in \Omega: y(\omega)<\lambda\}))$ then $y \in X$ and $\|x\|=\|y\|$.

Clearly the most natural examples of rearrangement invariant spaces are $L_{p}$ spaces for $1 \leq p \leq \infty$.

In our proof we will need a variant of a basically known Lemma which will serve as a tool to identify the right $p$.

LEMMA 1. Let $\varphi$ be a continuous increasing function on $[0,1]$ such that $\varphi(0)=0$ and for some $0<c \leq C<\infty$ and some $\Delta>1$ we have

$$
c \varphi\left(\Delta^{-n}\right) \varphi\left(\Delta^{n-N}\right) \leq \varphi\left(\Delta^{-N}\right) \leq C \varphi\left(\Delta^{n-N}\right) \varphi\left(\Delta^{-n}\right)
$$

for all $0 \leq n \leq N$. Then there exists $\alpha \geq 0$ and constants $0<a \leq A<\infty$ such that $a t^{\alpha} \leq \varphi(t) \leq A t^{\alpha}$ for all $t \in[0,1]$

Proof. We write $N=n+k$ and we get

$$
c \varphi\left(\Delta^{-n}\right) \varphi\left(\Delta^{-k}\right) \leq \varphi\left(\Delta^{-n-k}\right) \leq C \varphi\left(\Delta^{-k}\right) \varphi\left(\Delta^{-n}\right) .
$$

Inductively we get for arbitrary integer $s$

$$
c^{s} \varphi\left(\Delta^{-n}\right)^{s} \leq \varphi\left(\Delta^{-n s}\right) \leq C^{s} \varphi\left(\Delta^{-n}\right)^{s} .
$$

Taking logarithms with the base $\Delta$ we obtain

$$
s \log c+s \log \varphi\left(\Delta^{-n}\right) \leq \log \varphi\left(\Delta^{-n s}\right) \leq s \log C+s \log \varphi\left(\Delta^{-n}\right)
$$

so

$$
\left|\log \varphi\left(\Delta^{-n s}\right)-s \log \varphi\left(\Delta^{-n}\right)\right| \leq s M
$$


where $M=\max \{|\log c|,|\log C|\}$. This we rewrite as

$$
\left|\frac{\log \varphi\left(\Delta^{-n s}\right)}{n s}-\frac{\log \varphi\left(\Delta^{-n}\right)}{n}\right| \leq \frac{M}{n} .
$$

Thus for all natural $n$ and $m$ we obtain

$$
\begin{aligned}
& \left|\frac{\log \varphi\left(\Delta^{-m}\right)}{m}-\frac{\log \varphi\left(\Delta^{-n}\right)}{n}\right| \\
\leq & \left|\frac{\log \varphi\left(\Delta^{-m}\right)}{m}-\frac{\log \varphi\left(\Delta^{-m n}\right)}{m n}\right|+\left|\frac{\log \varphi\left(\Delta^{-m n}\right)}{m n}-\frac{\log \varphi\left(\Delta^{-n}\right)}{n}\right| \\
\leq & \frac{M}{m}+\frac{M}{n} .
\end{aligned}
$$

This implies that the $\operatorname{limit} \lim _{n \rightarrow \infty} \log \varphi\left(\Delta^{-n}\right) / n$ exists; we denote it by $\beta$. If we pass to the limit with $n \rightarrow \infty$ in inequality (2) we get

$$
\left|\frac{\log \varphi\left(\Delta^{-m}\right)}{m}-\beta\right| \leq \frac{M}{m}
$$

which we rewrite as

$$
\left|\log \frac{\varphi\left(\Delta^{-m}\right)}{\Delta^{\beta m}}\right| \leq M
$$

which gives

$$
c^{\prime} \Delta^{\beta m} \leq \varphi\left(\Delta^{-m}\right) \leq C^{\prime} \Delta^{\beta m}
$$

for $m=1,2, \ldots$. Since $\varphi$ is increasing we easily get

$$
a t^{-\beta} \leq \varphi(t) \leq A t^{-\beta} .
$$

Since clearly $\beta \leq 0$ we get the claim.

REMARK. Let us note that if the function $\varphi$ is defined on $[0, \infty)$ and (1) holds for all integers $N$ then $a t^{\alpha} \leq \varphi(t) \leq A t^{\alpha}$ for all $t>0$. To see this observe that by Lemma 1 we have $a t^{\alpha} \leq \varphi(t) \leq A t^{\alpha}$ for all $t \in[0,1]$ and applying (1) for $N=0$ and $n=1,2, \ldots$ we obtain $\varphi\left(\Delta^{-n}\right) \varphi\left(\Delta^{n}\right) \sim$ const. so $\varphi\left(\Delta^{n}\right) \sim\left(\Delta^{n}\right)^{\alpha}$. This shows our claim.

This Lemma is basically known, see [15] or [4, page 60].

We will be interested in the Haar system on $[0,1]^{d}$ which is defined as follows. First we define two functions on $\mathbb{R}$

$$
h^{0}(t)=\mathbf{1}_{[0,1]}= \begin{cases}1 & \text { when } t \in[0,1] \\ 0 & \text { otherwise }\end{cases}
$$

and

$$
h^{1}(t)=\mathbf{1}_{[0,1 / 2)}-\mathbf{1}_{[1 / 2,1]}= \begin{cases}1 & \text { when } t \in[0,1 / 2), \\ -1 & \text { when } t \in[1 / 2,1] \\ 0 & \text { otherwise }\end{cases}
$$

We define $E$ to be the set of all sequences $\varepsilon=\left(\varepsilon_{1}, \ldots, \varepsilon_{d}\right)$ where $\varepsilon_{i}=0,1$ and $\sum_{i=1}^{d} \varepsilon_{i}>0$. For $\varepsilon \in E$ we define

$$
h^{\varepsilon}\left(t_{1}, \ldots, t_{d}\right)=h^{\varepsilon_{1}}\left(t_{1}\right) \cdots \cdot h^{\varepsilon_{d}}\left(t_{d}\right) .
$$


The system $h_{n, k}^{\varepsilon}(t)=h^{\varepsilon}\left(2^{n} t-k\right)$ with $\varepsilon \in E, n \in \mathbb{Z}$ and $k \in \mathbb{Z}^{d}$ is a Haar wavelet basis in $\mathbb{R}^{d}$, normalised in $L_{\infty}$. The same system for $n=0,1,2, \ldots$ and $k \in L_{n}=$ $\left\{0,1, \ldots, 2^{n}-1\right\}^{d}$ and $\varepsilon \in E$ with the constant function added is a Haar system on $[0,1]^{d}$. The important fact about both those systems is that $\left|h_{n, k}^{\varepsilon}\right|$ is the characteristic function of a dyadic square of sidelength $2^{-n}$ so of measure $2^{-n d}$.

THEOREM 2. Let $X$ be a rearrangement invariant space on $[0,1]^{d}$. If the Haar system normalised in $X$ is a greedy basis in $X$ then $X=L_{p}[0,1]^{d}$ for some $1<p<\infty$ (with equivalent norm).

Proof. For $0 \leq t \leq 1$ we define $\varphi(t)=\left\|\mathbf{1}_{A}\right\|_{X}$ where $A \subset[0,1]^{d}$ is any set of measure $t$. Since $X$ is a rearrangement invariant space it is really a function of $t$; it is clearly an increasing function. Since $X$ cannot equal $L_{\infty}$ (which does not have any basis) we infer that $\varphi$ is continuous and $\varphi(0)=0$. We can assume that $\varphi(1)=1$.

We define $H_{n . k}^{\varepsilon}=\left(\varphi\left(2^{-n d}\right)\right)^{-1} h_{n, k}^{\varepsilon}$. It is a Haar system normalised in $X$. For each $\varepsilon \in E$ we have

$$
\left\|\sum_{k=0}^{2^{n d}-1} H_{n, k}^{\varepsilon}\right\|=\left\|\left(\varphi\left(2^{-n d}\right)\right)^{-1} \mathbf{1}_{[0,1]^{d}}\right\|=\left(\varphi\left(2^{-n d}\right)\right)^{-1} .
$$

Analogously for $N \geq n$ we have

$$
\left\|\sum_{k=0}^{2^{n d}-1} H_{N, k}^{\varepsilon}\right\|=\varphi\left(2^{(n-N) d}\right)\left(\varphi\left(2^{-N d}\right)\right)^{-1} .
$$

Since the Haar basis is democratic we get constants $0<c \leq C<\infty$ such that

$$
c \varphi\left(2^{(n-N) d}\right)\left(\varphi\left(2^{-N d}\right)\right)^{-1} \leq\left(\varphi\left(2^{-n d}\right)\right)^{-1} \leq C \varphi\left(2^{(n-N) d}\right)\left(\varphi\left(2^{-N d}\right)\right)^{-1}
$$

for all $n \leq N$. From Lemma 1 applied for $\Delta=2^{d}$ we infer that $\varphi(t) \sim t^{1 / p}$ for some $p>0$. Since $X$ is a Banach space, from the triangle inequality we get $1 \leq p<\infty$. We will show that $\|f\|_{X} \sim\|f\|_{p}$ for all $f \in X$. From the density argument it suffices to consider functions $f \geq 0$ of the form

$$
f=\sum_{j=1}^{N} a_{j} \mathbf{1}_{I_{j}}
$$

where $I_{j}$ are disjoint and $\left|I_{j}\right|=2^{-s d}$ for some $s \in \mathbb{N}$. For such an $f$ we can find $g$ of the form

$$
g=\sum_{j=1}^{N} 2^{k_{j} d / p} \mathbf{1}_{I_{j}}
$$

with $k_{j} \in \mathbb{Z}$ such that $g \leq f \leq 2^{d / p} g$. This implies that $\|g\|_{X} \leq\|f\|_{X} \leq 2^{d / p}\|g\|_{X}$ and $\|g\|_{p} \leq\|f\|_{p} \leq 2^{d / p}\|g\|_{p}$. So it suffices to check that $\|g\|_{X} \sim\|g\|_{p}$. Now let us define $K_{j}$ to be disjoint dyadic cubes of measure $2^{-s d}$. Since both $X$ and $L_{p}$ are rearrangement invariant we get $\|g\|=\left\|\sum_{j=1}^{N} 2^{k_{j} d / p} \mathbf{1}_{K_{j}}\right\|$ for both norm in $X$ and norm in $L_{p}$ By homogeneity we can additionally assume that $k_{j} \geq s$ for $j=1,2, \ldots, N$. Now we subdivide each cube $K_{j}$ into $2^{\left(k_{j}-s\right) d}$ equal dyadic cubes $K_{j, r}$ each of measure $2^{-k_{j} d}$. Let $\mathfrak{h}_{j, r}$ denote any Haar function $h_{k_{j}, k}^{\varepsilon}$ whose support equals $K_{j, r}$ multiplied by $2^{k_{j} d / p}$. Those functions 
are normalised in $L_{p}$ and seminormalised in $X$. We put

$$
x=\sum_{j=1}^{N} \sum_{r=1}^{2^{\left(k_{j}-s\right) d}} \mathfrak{h}_{j, r} .
$$

One easily checks that $|x|=|g|$ so $\|x\|_{X}=\|g\|_{X}$ and $\|x\|_{p}=\|g\|_{p}$. But $x$ is a sum of $M=\sum_{j=1}^{N} 2^{\left(k_{j}-s\right) d}$ seminormalised in $X$ Haar functions. Since the Haar basis is greedy in $X$ from lemma 1 and (3) we infer that $\|x\|_{X} \sim M^{1 / p}$. On the other hand

$$
\|x\|_{p}=\|g\|_{p}=\left(\sum_{j=1}^{N} 2^{k_{j} d}\left|I_{j}\right|\right)^{1 / p}=\left(\sum_{j=1}^{N} 2^{\left(k_{j}-s\right) d}\right)^{1 / p} \sim\|x\|_{X} .
$$

Thus we conclude that $\|\cdot\|_{X}$ and $\|\cdot\|_{p}$ are equivalent, which means that $X=L_{p}$ with equivalent norm. Since the Haar system is an unconditional basis in $X$, the case $p=1$ is not possible (it is known that the Haar system is not unconditional basis in $L_{1}$ and even that $L_{1}$ does not have any unconditional basis, see [4]), so we get $1<p<\infty$.

REMARK. Using the Remark after Lemma 1 the above proof shows that Theorem 2 also holds for symmetric spaces on $\mathbb{R}^{d}$.

REMARK. Observe that throughout most of this proof we use only the assumption that $\left\|\sum_{A} H_{n, k}^{\varepsilon}\right\| \sim\left\|\sum_{B} H_{n, k}^{\varepsilon}\right\|$ for sets $A$ and $B$ of Haar functions with disjoint supports and $|A|=|B|$. Natural modification of our argument shows that this assumption implies that $X=L_{p}$ with $1 \leq p<\infty$. Only to exclude $L_{1}$ we have to use the Haar functions with overlapping supports.

REMARK. If Haar is one democratic in $X$ on $[0,1]$ than $X=L_{2}[0,1]$ with equivalent norm. To see it observe that $\left\|h^{0}+h^{1}\right\|=\left\|21_{[0,1 / 2]}\right\|=2 \varphi(1 / 2)$ but also $=\| \varphi(1 / 2)^{-1} h_{1,0}+$ $\varphi(1 / 2)^{-1} h_{1,1} \|=\varphi(1 / 2)^{-1}$. Comparing we get $\varphi(1 / 2)=1 / \sqrt{2}$. From $(5)$ with $c=C=1$ we get inductively $\varphi\left(2^{-2}\right)=\varphi\left(2^{-1}\right)^{2}$ etc. so $\varphi\left(2^{-k}\right)=(\sqrt{2})^{-k}$ which gives $\varphi(t) \sim \sqrt{t}$.

Much attention was paid in recent years to wavelet bases on $\mathbb{R}$ and on $\mathbb{R}^{d}$. Let us recall (cf. [11], [2]) that a function $\Phi \in L_{2}(\mathbb{R})$ is a wavelet if the system $\Phi_{n, k}(x):=$ $2^{n / 2} \Phi\left(2^{n} x-k\right)$ for $n, k \in \mathbb{Z}$ (called a wavelet basis) is an orthonormal basis in $L_{2}(\mathbb{R})$. The Haar system is one of such bases corresponding to the Haar wavelet $h^{1}$. It is known (cf. $[11,2,12]$ ) that many natural wavelet bases are unconditional bases in $L_{p}(\mathbb{R})$ for $1<p<\infty$ equivalent to the Haar system (i.e. the map $\Phi_{n, k} \leftrightarrow h_{n, k}^{1}$ extends by linearity to an isomorphism of $\left.L_{p}(\mathbb{R})\right)$. We have

Proposition 1. If there exists a wavelet basis $\left(\Phi_{n, k}\right)_{n, k \in \mathbb{Z}}$ equivalent to the Haar basis in $L_{p}(\mathbb{R})$ for all $p, 1<p<\infty$ and greedy in a rearrangement invariant space $X$ on $\mathbb{R}$ then $X=L_{p}(\mathbb{R})$ for some $1<p<\infty$

Proof. We know from [7] that a greedy basis is unconditional so $X$ has an unconditional basis. This implies (see [9, Remark 9.6 and Theorem 1.e.4]) that the Haar system is unconditional in $X$ and $X$ is an interpolation space between $L_{p_{1}}(\mathbb{R})$ and $L_{p_{2}}(\mathbb{R})$ for some $1<p_{1}<p_{2}<\infty$. Since $\left(\Phi_{n, k}\right)_{n, k \in \mathbb{Z}}$ is equivalent to $\left(h_{n, k}^{1}\right)_{n, k \in \mathbb{Z}}$ in $L_{p_{1}}(\mathbb{R})$ and $L_{p_{2}}(\mathbb{R})$ we infer that $\left(\Phi_{n, k}\right)_{n, k \in \mathbb{Z}}$ is equivalent to $\left(h_{n, k}^{1}\right)_{n, k \in \mathbb{Z}}$ in $X$. This gives that the Haar system is greedy in $X$ so by Theorem 2 we get that $X=L_{p}(\mathbb{R})$ for some $p, 1<p<\infty$. 
REMARK. There exists a natural construction (wavelet tensor) of a wavelet basis on $\mathbb{R}^{d}$, see [11]. With obvious and trivial modification the above argument extends to the multivariate case.

3. Haar in Lorentz spaces. It is generally the case that the Haar system is the "best" basis in a rearrangement invariant space. It is true for example that if a rearrangement invariant space $X$ has an unconditional basis then the Haar system is such a basis. This suggests the conjecture that if a rearrangement invariant space $X$ has a greedy basis then the Haar system is greedy so by Theorem $2 X$ equals $L_{p}$ for some $1<p<\infty$. Unfortunately this is not true. There are examples of rearrangement invariant spaces with symmetric, so also greedy, basis, see [3]. Nevertheless we believe that "classical" rearrangement invariant spaces do not have greedy bases (unless they are $L_{p}$-spaces).

In this section we collect some remarks about Lorentz spaces. We are unable to show that $L_{p, q}$ spaces with $p \neq q$ do not have greedy basis (although we conjecture that this is the case) but our results below indicate that if such a basis exists it has to be very strange.

In this and subsequent sections we will consider only spaces on $[0,1]$ and the one dimensional Haar system $h_{n, k}^{1}$ which to avoid superscripts we will denote by $h_{n, k}$.

Let us first recall the definition and basic properties of a Lorentz space $L_{p, q}[0,1]$, $1 \leq p, q<\infty$. For a measurable function $f$ on $[0,1]$ by $f^{*}$ we denote its non-increasing rearrangement i.e. a non-increasing function on $[0,1]$ with the same distribution as $|f(x)|$. The space $L_{p, q}[0,1]$ is the collection of all (equivalence classes of) measurable functions $f$ on $[0,1]$ such that

$$
\|f\|_{p, q}=\left(\int_{0}^{1} f^{*}(x)^{q} x^{\frac{q}{p}-1} d x\right)^{1 / q}<\infty
$$

For $1 \leq q \leq p$ the quantity $\|\cdot\|_{p, q}$ is a norm but for $1<p<q$ the triangle inequality is not satisfied and $\|\cdot\|_{p, q}$ is only a quasi-norm. Nevertheless this quasi-norm is equivalent to the norm. It is also clear that $L_{p, p}=L_{p}$. We have the following continuous embeddings:

$$
L_{p_{1}, q_{1}}[0,1] \subset L_{p_{1}, q_{2}}[0,1] \subset L_{p_{2}, q_{3}}[0,1]
$$

whenever $q_{1} \leq q_{2}$ and $p_{1}>p_{2}$. It is also known that $L_{p, q}[0,1]$ has an unconditional basis only when $1<p<\infty$ and $1 \leq q<\infty$. In such situation the Haar system is an unconditional basis.

First we want to check what subsequences of the Haar system are greedy in its span in $L_{p, q}$ norm.

It follows from Proposition 8.10 from [3] and Lemma 2.1 from [1] that any unconditional basis in $L_{p, q}[0,1], 1<p<\infty, 1 \leq q<\infty$ has a subsequence equivalent to the unit vector basis in $\ell_{q}$. So we have

Lemma 2. If $\left(x_{n}\right)_{n=1}^{\infty}$ is a greedy basis in $L_{p, q}[0,1], 1<p<\infty, 1 \leq q<\infty$ then $\left\|\sum_{n \in A} x_{n}\right\|_{p, q} \sim|A|^{1 / q}$.

Our next aim is to exhibit such subsequences in the Haar system.

Proposition 2. Let $x_{n}=2^{n / p} h_{n, k(n)}$ for $n=1,2, \ldots$ and $0 \leq k(n)<2^{n}$. The sequence $\left(x_{n}\right)_{n=1}^{\infty}$ is in $L_{p, q}[0,1]$ equivalent to the unit vector basis in $\ell_{q}$. 
Proof. Let us fix a subsequence of the Haar system of the form $h_{n, l(n)}$ for $n=1,2, \ldots$ in such a way that $\operatorname{supp} h_{n, l(n)}=\left(2^{-n}, 2^{-n+1}\right)$ so those Haar functions have disjoint supports. Using this sequence we define operators $Q$ and $S$ on the Haar system as follows

$$
Q\left(h_{n, j}\right)= \begin{cases}h_{n, l(n)} & \text { if }(n, j)=(n, k(n)), \\ 0 & \text { otherwise }\end{cases}
$$

and

$$
S\left(h_{n, j}\right)= \begin{cases}h_{n, k(n)} & \text { if }(n, j)=(n, l(n)), \\ 0 & \text { otherwise. }\end{cases}
$$

One easily checks and it is well known that both operators extend to continuous linear operators on $L_{p}[0,1]$ for $1<p<\infty$ so by interpolation they are continuous on $L_{p, q}[0,1]$. This means that the sequence $\left(x_{n}\right)_{n=1}^{\infty}$ is equivalent to the sequence $2^{n / p} h_{n, l(n)}$. In other words we can assume that $x_{n}$ has disjoint supports. For $q \leq p$ we have (below $\tau$ denotes the measure preserving transformation on $[0,1]$ )

$$
\begin{aligned}
\left\|\sum_{n=1}^{\infty} a_{n} x_{n}\right\|_{p, q}^{q} & \leq\left\|\sum_{n=1}^{\infty} a_{n} 2^{n / p} h_{n, l(n)}\right\|_{p, q}^{q} \\
& =\sup _{\tau} \int_{0}^{1}\left|\sum_{n=1}^{\infty} a_{n} 2^{n / p} h_{n, l(n)}(\tau(t))\right|^{q} t^{\frac{q}{p}-1} d t \\
& =\sup _{\tau} \sum_{n=1}^{\infty}\left|a_{n}\right|^{q} \int_{0}^{1}\left|2^{n / p} h_{n, l(n))}(\tau(t))\right|^{q} t^{\frac{q}{p}-1} d t \\
& \leq \sum_{n=1}^{\infty}\left|a_{n}\right|^{q} \sup _{n} \int_{0}^{2^{-n}} 2^{n q / p} t^{\frac{q}{p}-1} d t \\
& =\sum_{n=1}^{\infty}\left|a_{n}\right|^{q} .
\end{aligned}
$$

On the other hand we have

$$
\left\|\sum_{n=1}^{\infty} a_{n} x_{n}\right\|_{p, q}^{q} \geq \sum_{n=1}^{\infty}\left|a_{n}\right|^{q} 2^{n q / p} \int_{2^{-n}}^{2^{-n+1}} t^{\frac{q}{p}-1} d t \geq C \sum_{n=1}^{\infty}\left|a_{n}\right|^{q} .
$$

The case $q>p$ follows by duality.

Lemma 3. Let $0<j_{1}<j_{2}<\cdots<j_{s} \leq 2^{n}$ be a sequence of integers. Then

$$
\left\|\sum_{i=1}^{s} 2^{n / p} h_{n, j_{i}}\right\|_{p, q}=\left(\frac{p}{q}\right)^{1 / q} s^{1 / p} .
$$

Proof. We have

$$
\begin{aligned}
\left\|\sum_{i=1}^{s} 2^{n / p} h_{n, j_{i}}\right\|_{p, q} & =2^{n / p}\left\|\mathbf{1}_{\left[0, s 2^{-n}\right]}\right\|_{p, q}=2^{n / p}\left(\int_{0}^{s 2^{-n}} t^{\frac{q}{p}-1} d t\right)^{1 / q} \\
& =2^{n / p}\left(\frac{p}{q}\left(s 2^{-n}\right)^{q}\right)^{1 / q}=\left(\frac{p}{q}\right)^{1 / q} s^{1 / p}
\end{aligned}
$$

From the above we obtain 
THEOREM 3. If a subsequence of the Haar system is greedy (in its linear span) in $L_{p, q}[0,1]$ with $p \neq q, 1<p<\infty$ and $1 \leq q<\infty$, then it is equivalent to the unit vector basis in $\ell_{q}$. Proof. From Proposition 2 we see that our subsequence of the Haar system has a subsequence equivalent to the unit vector basis in $\ell_{q}$ so by democracy the sum of any $N$ elements of our subsequence has the norm $\sim N^{1 / q}$. If we compare this with Lemma 3 we infer that there exists a natural number $s$ such that for each $n$ our subsequence contains at most $s$ elements from each level of Haar functions $\left(h_{n, j}\right)_{j=0}^{2^{n}-1}$. Applying Proposition 2 once more we see that our subsequence is equivalent to the unit vector basis in $\ell_{q}$.

Now we will define generalised Haar systems on $[0,1]$. Such systems were studied e.g. in [6]. Let $\mathcal{T}=\left\{t_{j}\right\}_{j=0}^{\infty}$ be a sequence of distinct points from the interval [0,1] which is dense in $[0,1]$ and such that $t_{0}=0$ and $t_{1}=1$. By $l\left(t_{n}\right)$ we mean the biggest of those points $t_{0}, t_{1}, \ldots, t_{n-1}$ which are $<t_{n}$ and by $r\left(t_{n}\right)$ we mean the smallest of those points $t_{0}, t_{1}, \ldots, t_{n-1}$ which are $>t_{n}$. The generalised Haar system corresponding to the sequence $\mathcal{T}$ is defined as follows: $h_{1}(t)=1$ and for $n>1$ we put

$$
h_{n}(t)= \begin{cases}0 & \text { if } t \leq l\left(t_{n}\right) \text { or } t \geq r\left(t_{n}\right), \\ \left(t_{n}-l\left(t_{n}\right)\right)^{-1} & \text { if } t \in\left(l\left(t_{n}\right), t_{n}\right), \\ -\left(r\left(t_{n}\right)-t_{n}\right)^{-1} & \text { if } t \in\left(t_{n}, r\left(t_{n}\right)\right) .\end{cases}
$$

It is known that each generalised Haar system is an unconditional and greedy basis in $L_{p}[0,1]$ for $1<p<\infty$, see [6].

Corollary 1. No generalised Haar system is greedy in $L_{p, q}[0,1] 1<p<\infty$ and $1 \leq$ $q<\infty, p \neq q$.

Proof. It was shown in [6, Theorem 3.2] that each generalised Haar system is in $L_{p}$ equivalent to a subsequence of the dyadic Haar system. The argument given there shows that the map which establishes the equivalence is the same for all $p$ 's with $1<p<\infty$. By interpolation it implies that the generalised Haar system in $L_{p, q}[0,1], 1<p<\infty$ and $1 \leq q<\infty, p \neq q$ is equivalent to a subsequence of the dyadic Haar system in $L_{p, q}[0,1]$. By Theorem 3 it cannot be greedy because $L_{p, q}$ is not isomorphic to $\ell_{q}$.

4. Greedy bases in Lorentz spaces. In this section we present some observations which suggest that if there exists a greedy basis in $L_{p, q}[0,1]$ with $p \neq q$ than it has to be rather strange. Since our results are highly nonconclusive we present them only for the simplest choice of parameters $p, q$. It is clear that our Propositions 3 and 4 hold for some other choices of parameters.

Proposition 3. No orthogonal system is a greedy basis in $L_{2, q}[0,1]$ for $1 \leq q<\infty$, $q \neq 2$.

LEMMA 4. Let $\left(e_{n}\right)_{n=1}^{\infty}$ be a greedy basis in a Banach space $X$ such that $\left\|\sum_{n \in A} e_{n}\right\| \sim$ $|A|^{1 / p}$ for some $p, 1 \leq p<\infty$. Then there are constants $0<c \leq C$ such that

$$
c\left\|\left(a_{n}\right)\right\|_{p, \infty} \leq\left\|\sum_{n=1}^{\infty} a_{n} e_{n}\right\| \leq C\left\|\left(a_{n}\right)\right\|_{p, 1}
$$

for all sequences of scalars. 
This Lemma is basically known (see [8, Theorem 2.5.2] or [13, Theorem 3]). Since I was unable to find an exact reference the proof is given for the convenience of the reader.

Proof. Let us assume (to simplify the notation) that $\left|a_{n}\right| \searrow 0$ and put $n_{k}=\mid\left\{n:\left|a_{n}\right|>\right.$ $\left.2^{-k}\right\} \mid$. The right hand side inequality for $p=1$ is obvious. For $p>1$ we have

$$
\begin{aligned}
\left\|\sum_{n=1}^{\infty} a_{n} e_{n}\right\| & \leq C \sum_{k} 2^{-k-1}\left\|\sum_{n_{k}+1}^{n_{k+1}} e_{n}\right\| \\
& \leq C \sum_{k} 2^{-k} n_{k}^{1 / p} \leq C \sum_{k} 2^{-k} \sum_{s=1}^{n_{k}} s^{\frac{1}{p}-1} \\
& =C \sum_{s=1}^{\infty} s^{\frac{1}{p}-1} \sum_{k: n_{k}>s} 2^{-k} \leq C \sum_{s=1}^{\infty} s^{\frac{1}{p}-1}\left|a_{s}\right| \\
& \leq C\left\|\left(a_{n}\right)\right\|_{p, 1} .
\end{aligned}
$$

On the other hand for each $N$

$$
\left\|\sum_{n} a_{n} e_{n}\right\| \geq C\left\|a_{N} \sum_{n=1}^{N} e_{n}\right\| \geq C\left|a_{N}\right| N^{1 / p}
$$

so $\left\|\left(a_{n}\right)\right\|_{p, \infty} \leq C\left\|\sum_{n} a_{n} e_{n}\right\|$.

Now we are ready for the proof of the Proposition. Let $\left(f_{n}\right)_{n=1}^{\infty}$ be an orthogonal system which is a greedy basis in $L_{2, q}[0,1]$ and $\left\|f_{n}\right\|_{2, q}=1$ for $n=1,2, \ldots$ Let us start with the case $1 \leq q<2$ and fix $r$ such that $q<r<2$. We have the commutative diagram

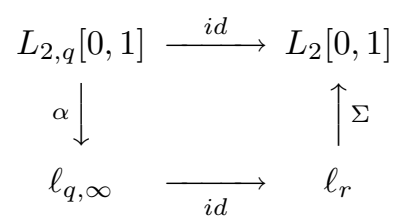

where $i d$ denotes the identity embedding. For $f=\sum_{n} a_{n} f_{n}$ we put $\alpha(f)=\left(a_{n}\right)_{n=1}^{\infty}$. By Lemma $4 \alpha$ is a continuous operator. We define $\Sigma\left(\left(\xi_{n}\right)_{n=1}^{\infty}\right)=\sum_{n=1}^{\infty} \xi_{n} f_{n}$. It is also a continuous operator because

$$
\begin{aligned}
\left\|\sum_{n=1}^{\infty} \xi_{n} f_{n}\right\|_{2} & =\sqrt{\sum_{n=1}^{\infty}\left|\xi_{n}\right|^{2}\left\|f_{n}\right\|_{2}^{2}} \leq C \sqrt{\sum_{n=1}^{\infty}\left|\xi_{n}\right|^{2}\left\|f_{n}\right\|_{2, q}^{2}} \\
& \leq C \sqrt{\sum_{n=1}^{\infty}\left|\xi_{n}\right|^{2}} \leq C\left(\sum_{n=1}^{\infty}\left|\xi_{n}\right|^{r}\right)^{1 / r} .
\end{aligned}
$$

Clearly $i d=\Sigma \circ i d \circ \alpha$ so it is a commutative diagram. This however is impossible. It is a well known direct consequence from the Khintchin inequality that $i d: L_{2, q}[0,1] \rightarrow L_{2}[0,1]$ is an isomorphism when restricted to the infinite dimensional Hilbert space $R$ spanned by the Rademacher functions. This would imply that $i d \circ \alpha(R)$ is a subspace of $\ell_{r}$ isomorphic to a Hilbert space but it is known that no subspace of $\ell_{r}$ is isomorphic to an infinite dimensional Hilbert space. This contradiction proves our claim. 
For the case $q>2$ we choose $r$ such that $q>r>2$ and consider a dual diagram

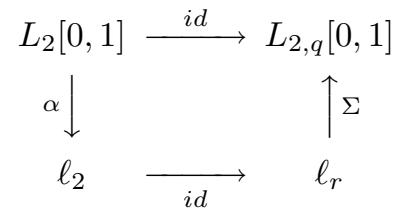

where $\alpha$ and $\Sigma$ are defined by the same formulas. Analogously we obtain that they are continuous and that the diagram is commutative. This leads to the contradiction exactly as before.

Before we proceed let us introduce some notation. For given $N=1,2, \ldots$ let $I_{n}=$ $\left[\frac{n-1}{N}, \frac{n}{N}\right)$ for $n=1,2, \ldots, N$. By $\mathcal{C}_{N}$ we denote the space of all function of the form $f=\sum_{n=1}^{N} a_{n} \mathbf{1}_{I_{n}}$.

Lemma 5. For a function $f \in \mathcal{C}_{N}$ and $1<p<\infty$ we have

$$
C(\log N)^{\frac{1}{p}-\frac{1}{q}}\|f\|_{p, q} \leq\|f\|_{p} \leq\|f\|_{p, q} \quad \text { if } q<p
$$

and

$$
\|f\|_{p, q} \leq\|f\|_{p} \leq C(\log N)^{\frac{1}{p}-\frac{1}{q}}\|f\|_{p, q} \quad \text { if } q>p
$$

where constants do not depend on $f$ and $N$.

Proof. Clearly $\|f\|_{p}=N^{-1 / p}\left(\sum_{n=1}^{N}\left|a_{n}\right|^{p}\right)^{1 / p}$ and easy calculation shows that

$$
\|f\|_{p, q} \sim N^{-1 / p}\left(\sum_{n=1}^{N}\left|a_{n}^{*}\right|^{q} n^{\frac{q}{p}-1}\right)^{1 / p}
$$

where as usual $\left|a_{n}^{*}\right|$ denotes the nonincreasing rearrangement of $\left(\left|a_{n}\right|\right)_{n=1}^{N}$. For $q<p$ we use Hölder's inequality to obtain

$$
\begin{aligned}
\sum_{n=1}^{N}\left|a_{n}^{*}\right|{ }^{q} n^{\frac{q}{p}-1} & \leq\left(\sum_{n=1}^{N}\left|a_{n}^{*}\right|^{p}\right)^{q / p}\left(\sum_{n=1}^{N} n^{\left(\frac{q}{p}-1\right) \frac{p}{p-q}}\right)^{\frac{p-q}{p}} \\
& =\|f\|_{p}^{q}\left(\sum_{n=1}^{N} n^{-1}\right)^{\frac{p-q}{p}} \leq C\|f\|_{p}^{q}(\log N)^{\frac{p-q}{p}} .
\end{aligned}
$$

From (13) and (14) we get (11). The inequality (12) is proved analogously.

Now we are ready to prove our last observation.

Proposition 4. Let $\left(f_{n}\right)_{n=1}^{\infty}$ be a greedy basis in $L_{2, q}[0,1]$. There exists a constant $C>0$ such that if for some $k$ and each $n=1,2, \ldots, k$ we have $f_{n} \in \mathcal{C}_{N(k)}$, then $N(k) \geq \exp k / C$.

Proof. We will consider only the case $q<2$, the case $q>2$ is proved analogously. From Lemma 2 we infer that $\left\|\sum_{n=1}^{k} \pm f_{n}\right\|_{2, q} \sim k^{1 / q}$. It is a well known consequence of the Khintchine inequality that for any $g_{n}$ in $L_{2}$ the average over all signs of $\left\|\sum_{n} \pm g_{n}\right\|_{2}=$ $\sqrt{\sum_{n}\left\|g_{n}\right\|_{2}^{2}}$. So there exists a choice of signs such that $\left\|\sum_{n=1}^{k} \pm f_{n}\right\|_{2} \leq \sqrt{\sum_{n=1}^{k}\left\|f_{n}\right\|_{2}^{2}}$. 
For this choice of signs from (11) we get

$$
\begin{aligned}
k^{1 / q} & \leq C(\ln N(k))^{\frac{1}{q}-\frac{1}{2}} \sqrt{\sum_{n=1}^{k}\left\|f_{n}\right\|_{2}^{2}} \\
& \leq C(\ln N(k))^{\frac{1}{q}-\frac{1}{2}} \sqrt{\sum_{n=1}^{k}\left\|f_{n}\right\|_{2, q}^{2}} \\
& =C k^{1 / 2}(\ln N(k))^{\frac{1}{q}-\frac{1}{2}} .
\end{aligned}
$$

This immediately gives the claim.

REMARK. The above arguments carry over to the situation when $\mathcal{C}_{N}$ is a subspace generated by characteristic function of a partition of $[0,1]$ into $N$ sets of measure $\sim N^{-1}$. This in particular implies that if a greedy basis consists of linear combinations of Haar functions, then in the best case we need approximately $n$ levels of the Haar functions to write first $n$ elements of the basis.

\section{References}

[1] N. L. Carothers and S. J. Dilworth, Subspaces of $L_{p, q}$, Proc. Amer. Math. Soc. 104 (1988), $537-545$.

[2] I. Daubechies, Ten Lectures on Wavelets, SIAM, Philadelphia, 1992.

[3] W. B. Johnson, B. Maurey, G. Schechtman and L. Tzafriri, Symmetric structures in Banach spaces, Mem. Amer. Math. Soc. 217 (1979).

[4] J. Lindenstrauss and L. Tzafriri, Classical Banach Spaces I, Springer, Berlin, 1977.

[5] J. Lindenstrauss and L. Tzafriri, Classical Banach Spaces II, Springer, Berlin, 1979.

[6] A. Kamont, General Haar systems and greedy approximation, Studia Mathematica 145 (2001), 165-184.

[7] S. V. Konyagin and V. N. Temlyakov, A remark on greedy approximation in Banach spaces, East J. Approx. 5 (1999), 1-15.

[8] S. G. Krein, Yu. I. Petunin and E. M. Semenov, Interpolation of Linear Operators, Nauka, Moscow, 1978 (in Russian) or English translation, Amer. Math. Soc., Providence, 1982.

[9] I. Novikov and E. Semenov, Haar Series and Linear Operators, Kluwer Academic Publishers, Dordrecht, 1997.

[10] V. N. Temlyakov, The best m-term approximation and greedy algorithms, Adv. Comput. Math. 8 (1998), 249-265.

[11] P. Wojtaszczyk, A Mathematical Introduction to Wavelets, London Math. Soc. Student Texts 37, Cambridge University Press, Cambridge, 1997.

[12] P. Wojtaszczyk, Wavelets as unconditional bases in $L_{p}(\mathbb{R})$, J. Fourier Anal. Appl. 5 (1999), 73-85.

[13] P. Wojtaszczyk, Greedy algorithm for general biorthogonal systems, J. Approx. Theory 107 (2000), 293-314.

[14] P. Wojtaszczyk, Greedy type bases in Banach spaces, in: Constructive Theory of Functions, B. Bojanov (ed.), Darba, Sofia, 2003, 136-155.

[15] M. Zippin, On perfectly homogeneous bases in Banach spaces, Israel J. Math. 4 (1966), 265-272. 